\title{
THE SKILLING INDIAN SCIENTISTS FOR LAST OVER FIVE DECADES
}

Indian Drug Manufacturers' Association (IDMA) was formed in 1961, exactly 56 years ago. It started publishing "Indian Drugs" a publication devoted to various aspects, amongst which were "giving space for Indian Pharmaceutical Scientists and others to find a place to publish the results of their research". Indian Drugs - monthly scientific and technical journal has completed its Golden Jubilee last year and is now in its 53rd year. Indian Drugs is also an online version with dedicated website www.indiandrugsonline.org apart from bringing out the Print version still.

Scientific writing is more a skill and an art rather than just writing. Doing scientific

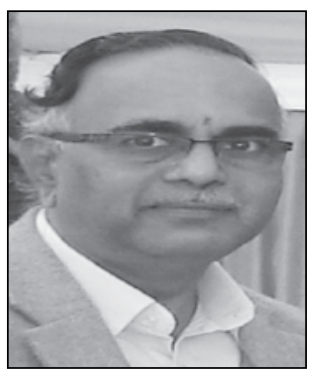
research is purely "Science" and no art. During the 60's that Indian pharmacy schools and other schools of science were learning to do research. Research methodologies have themselves, undergone sea changes. From many tests to be done manually, innovations in analytical technologies, measurement sciences, tools of measurement supported by electronics/image analysis/ biochemical analytical techniques/molecular analysis/ bio-markers and, above all, use of IT tools have transformed the area of scientific research. Yet, during the period of 60 s to the beginning of the new millennium, pharmacy colleges have promoted mandatorily research as part of the Master's degree courses and in Doctoral research. The fact that research done by young students for their Master's degree were limited by resources and time of may be 6-12 months are well known. It can be easily said that such research was more a training/skill development education to do research, rather than really high quality and impactful outcome bringing research. However, it produced some remarkable outcomes, many of which were of use to the developing pharma industry and regulators. Education and providing on-the-job research training were the main motives. Due to various reasons that need not be listed here, publishing the results of such research still was difficult. Indian Drugs provided the route and space.

The vision of the founders IDMA is still being carried by Indian Drugs. In many Editorial Board meetings of Indian Drugs, often the discussions have been on the "standard and impact" of the papers that appear in this journal. Most of us who serve on this board in honorary capacity still are passionate to stick to the original objective-that Indian Drugs should provide a platform to publish the results of young researchers of India and continue to do so.

Changing scenario of being “Impact Factor centric", predatory journals appearing in 100's, indexation of publications in peer-reviewed data bases, fear of plagiarism, need for payment of page charges, conversion of research publications into an industry/income generation(?) outlet, and firms beginning to invest in developing IT tools to manage the entire aspects of the publication from the time a paper is submitted till it is published or rejected, and other aspects have not daunted Indian Drugs. These are being taken into consideration and strides have been made where necessary. Online publication of Indian Drugs through a locally developed IT tool/website is one such stride.

Indian Drugs and IDMA have on and off conducted seminars/workshops and meetings on research methodologies, scientific writing skills development and related subjects. How to write a scientific paper, how to read a research paper and how to write a research proposal are very important, as important as doing the research. Being a reviewer for more than 3 decades for many journals, research project proposals submitted to national financial granting bodies for research, there is scope for constant improvement in the same. Most of the writing is still not the best writing. It is the faculty of the teaching institutions that need to impart these skills and keep doing so. Indian Drugs and IDMA can only play a supporting role-which it is doing and has done for over 5 decades.

Indian Drugs and IDMA are now sitting on a treasure of scientific research publications, with most of it being from Indian Laboratories. Today scientists have been made-shall we say "lazy" and who want to get every information by the click of the button. Gone are those days when one used to sit and make notes, write out the Chemical Index abstract numbers and briefs, before going to look for the dusty bound 
volumes of the Chemical Abstracts, for that matter Biological/Analytical abstracts etc for literature search, A timeconsuming hard work. Digitization is the mantra, and obviously it is to be taken up by Indian Drugs and IDMA to digitize the contents of all the Volumes of Indian Drugs for the past 53 years. The Credit for all this is to be given to IDMA and, more importantly Dr. Abraham Patani, the founding Editor of Indian Drugs who has weathered many a stormall these years in making this happen, and more importantly, preserve these Volumes of Indian Drugs since its inception. His dedication is unparalleled and a true representation of the patience and commitment to this cause. Even at the cost of self-praising, IDMA and its staff has over the long years provided the necessary support and this needs to be lauded. The members of the Editorial Board have stood by the ethos needed, and by constant and regular interventions, have upgraded the "Standard" of papers published in Indian Drugs by rejecting more papers and guiding improvements in reporting skills when the data were good and writing were poor. Today, the Journal also does a quick check on the data and integrity (read plagiarism) before taking up review using tools available. Starting to publish an Editorial is yet another change and this would be the medium through which researchers will get more information.

Skilling India is an objective, and the term coined and made into a national programme in the last 3 years by the Government. Indian Drugs and IDMA has done it for over 53 years by - Recognizing (Indian research and giving space for it) -Recording (publication in Indian Drugs) \& Rewarding (giving awards for best papers).

The journey needs to continue....

D B Anantha Narayana, Bengaluru. Drandba50@gmail.com

About The Member, Editorial Advisory Board
Dr Ananatha Narayana is an Eminent Pharmacist, who taught Pharmacy for 10 years and has worked in
Ranbaxy Labs, Dabur Research Foundation and Unilever Research (India) - in a span of over 40 years of
contribution to Indian Pharmaceutical industry and research. He superannuated in 2010 from Unilever, but
continues to serve the nation and profession through his membership in Indian Pharmacopeia Commission's
scientific body, Food Safety \& Standards Authorities Scientific Panels and Working Groups. He has championed
the roel of Phytopharmaceuticals as Drugs and shaped Functional Foods, Supplements and Nutraceutical
Regulations being notified by the Government of India. He is currently Chief Scientific officer of the Ayurvidye
Trust, Bengaluru.

